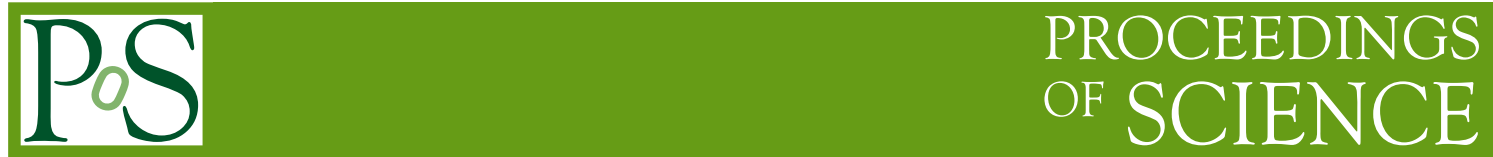

\title{
Local chiral fermions
}

\author{
Michael Creutz ${ }^{* \dagger}$ \\ Brookhaven National Laboratory \\ Upton, NY 11973, USA \\ E-mail: creutz@bnl.gov
}

Borici's construction of minimally doubled chiral fermions builds on a linear combination of two unitarily related naive fermion actions. Being strictly local, extremely efficient numerical implementation should be possible. The resulting system is symmetric under the subgroup of the hypercubic group that preserves a major hypercube diagonal. The symmetry includes both parity even and odd transformations, but allows for an anisotropy to appear at finite lattice spacing.

The XXVI International Symposium on Lattice Field Theory

July 14 - 19, 2008

Williamsburg, Virginia, USA

*Speaker.

$\dagger$ This manuscript has been authored under contract number DE-AC02-98CH10886 with the U.S. Department of Energy. Accordingly, the U.S. Government retains a non-exclusive, royalty-free license to publish or reproduce the published form of this contribution, or allow others to do so, for U.S. Government purposes. 


\section{Introduction}

I describe a strictly local fermion Dirac operator $\mathscr{D}(A)$ with an exact chiral symmetry manifested in anti-commutation with $\gamma_{5}$, i.e. $\gamma_{5} \mathscr{D}=-\mathscr{D} \gamma_{5}$. By strictly local I mean that only nearest neighbor terms appear in the fermion action. The operator describes two fermion flavors, the minimum required for chiral symmetry to exist. I develop this action as a linear combination of two "naive" fermion actions, following a line of reasoning similar to that presented by Borici [1].

The theory is not symmetric under the full hyper-cubic group, but the subgroup thereof that preserves one fixed direction up to a sign. These symmetries include transformations of both even and odd parity. On renormalization, interactions can introduce a lattice anisotropy at finite cutoff.

\section{Doubling and chiral symmetry}

Spontaneously broken chiral symmetry is fundamental to our understanding of low energy hadronic physics. Pions are elegantly described as quantum mechanical waves propagating through a background quark condensate. In addition, chiral symmetry provides powerful tools enabling extrapolations to the physical quark masses from the heavier values currently practical in lattice gauge simulations.

These issues are deeply entangled with quantum mechanical anomalies that eliminate one symmetry of the classical Lagrangian. With $N_{f}$ flavors of quark, the naive axial $U\left(N_{f}\right)$ is reduced to $S U\left(N_{f}\right)$. With only one flavor, all chiral symmetry is removed; so, multiple flavors are necessary for chiral symmetry to be relevant. Nielsen and Ninomiya [2] have given a formal topological argument that any lattice action with chiral symmetry must describe at least two flavors.

If one ignores the anomaly and writes a simple lattice action that is chirally symmetric, something must go wrong. The usual result is that the fermion field describes multiple species, and the extra species cancel the anomalies. The most naive discretization, which will play an essential role below, involves 16 species in four dimensions. Staggered fermions divide out an exact $S U(4)$ symmetry of the naive formulation to reduce the multiplicity to four [3]. The Wilson fermion [4] approach successfully removes all doublers at the expense of breaking all chiral symmetries. Elegant newer approaches based on perfect actions [5], domain walls in five dimensions [6], or the overlap operator [7] do maintain much of the desired chiral symmetry with arbitrary $N_{f}$, but involve computationally expensive non-local actions. Also, these approaches tend to obscure the anomaly; for example, with the overlap one introduces a gauge field dependent matrix $\hat{\gamma}_{5}$, the trace of which gives the winding number of a given gauge configuration.

The Nielsen-Ninomiya theorem requires any chirally symmetric lattice action to describe at least two species. Actions which satisfy the minimal doubling of just two have been known for some time [8,9], and have recently stimulated new interest $[1,10,11,12]$. There are a variety of compelling reasons to revisit these actions. First is the failure of the rooting procedure popularly used to reduce the doubling of staggered fermions [13]. Second is the lack of an exact chiral symmetry for Wilson fermions, complicating extrapolations to physical fermion masses. And third is the severe computational demands of the domain-wall and overlap approaches. 

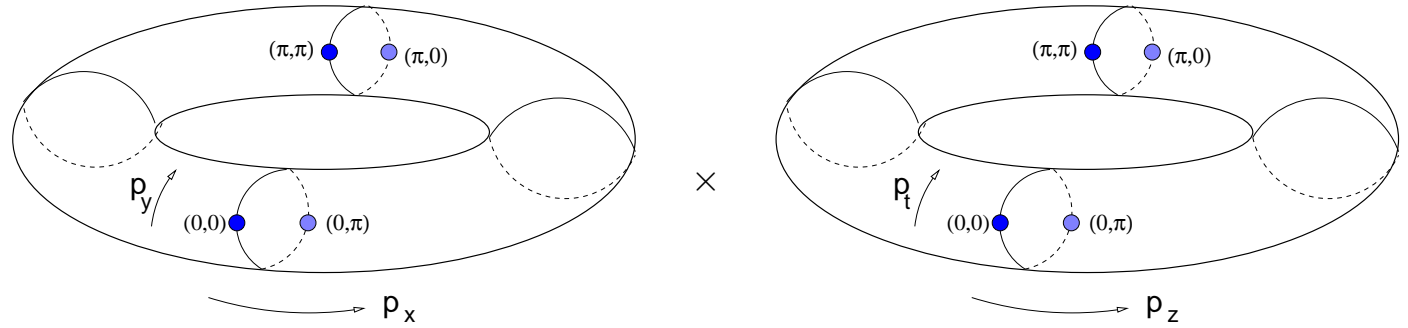

Figure 1: Representing momentum space as a product of toroids, a massless Dirac equation can be obtained from naive fermions by expanding about any point where the components of momentum are either 0 or $\pi$. This gives rise to the 16 doublers of the approach.

Here I construct a minimally doubled fermion action closely following Borici's formalism [1]. I use a linear combination of two unitarily equivalent naive fermion actions. This combination will be crafted so that only two of the original 16 doublers for each action survive.

\section{Naive fermions}

The so called "naive fermion" approach plays a crucial role in the following; so, I begin by reviewing this action. Work with a conventional hyper-cubic lattice with gauge fields implemented as group elements on the links. The details of the gauge part of the action play no role here. When a fermion hops in a forward direction $\mu$ between neighboring sites, it picks up a factor of $\gamma_{\mu} U$. Here $\gamma_{\mu}$ is the usual Hermitean Dirac matrix while $U$ represents the gauge field on the corresponding link. For the reverse, or backward, hop on the same link, the contribution is $-\gamma_{\mu} U^{\dagger}$. The minus sign makes the fermion operator an anti-Hermitean matrix when viewed as a matrix in the direct product of the site space with the internal symmetry and spinor spaces. I work here with the convention of a unit hopping parameter; for the massless case any other hopping parameter can be scaled away with a redefinition of the fields. I also work in lattice units so that all site coordinates are integers and the lattice spacing does not appear explicitly.

As is well known, this action describes the physics of 16 fermion species, frequently referred to as doublers. The Dirac operator, $D$, anti-commutes with $\gamma_{5}$. This represents an exact chiral symmetry. Because the doublers use different effective gamma matrices, half of them rotate in each direction under a chiral rotation. Thus this is a non-singlet chiral symmetry.

In the free field limit where all the link matrices are the identity, this theory is easily solved in momentum space. The Dirac operator factors into independent pieces for each momentum $p$, taking the form

$$
D(p)=2 i \sum_{\mu} \gamma_{\mu} \sin \left(p_{\mu}\right)
$$

Expanding about small momentum gives the usual Dirac behavior $D(p) \simeq 2 i \sum_{\mu} \gamma_{\mu} p_{\mu}$. The doublers appear on expanding not about zero momentum but around points where some components of $p$ are approximately $\pi$. Thus, in addition to the excitations around $p_{\mu}=0$, there are 15 other points in momentum space where the action is small. Visualizing momentum space as a direct product of two toroids, the zeros occur as sketched in Fig. (1). 


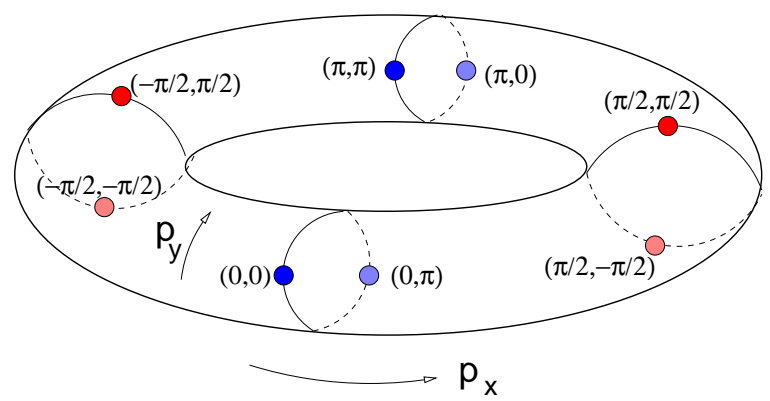

Figure 2: The points in momentum space furthest from those giving the Dirac equation are located at $p_{\mu}= \pm \pi / 2$. Our second naive fermion action will have its zeros at these points.

\section{A unitary transformation}

I now do a transformation on the above action to generate a superficially different but physically equivalent naive fermion action. Begin by considering maximally distant momenta from the zeros of $D$. There are 16 such points, occurring whenever the components of the momentum satisfy $p_{\mu}= \pm \pi / 2$. These points are sketched in Fig. (2) for the $x, y$ sub-torus. Arbitrarily select one of these points; here I consider $p_{\mu}=+\pi / 2$ for all $\mu$. Here the original action becomes

$$
D\left(p_{\mu}=\pi / 2\right)=2 i \sum_{\mu} \gamma_{\mu}=4 i \Gamma
$$

where I define the quantity

$$
\Gamma \equiv \frac{1}{2}\left(\gamma_{1}+\gamma_{2}+\gamma_{3}+\gamma_{4}\right)
$$

This is a unitary, Hermitean, and traceless four by four matrix in spinor space.

Now consider a unitary transformation on the original fields

$$
\begin{aligned}
& \psi^{\prime}=e^{-i \pi\left(x_{1}+x_{2}+x_{3}+x_{4}\right) / 2} \Gamma \psi \\
& \bar{\psi}^{\prime}=e^{i \pi\left(x_{1}+x_{2}+x_{3}+x_{4}\right) / 2} \bar{\psi} \Gamma .
\end{aligned}
$$

Here the $x_{\mu}$ are the integer coordinates of the lattice. The phases move the zeros in momentum space from $p_{\mu}=0, \pi$ to the maximally distant points $p_{\mu}= \pm \pi / 2$. The factors of $\Gamma$ modify the gamma matrices for the new action to

$$
\gamma_{\mu}^{\prime}=\Gamma \gamma_{\mu} \Gamma
$$

Note that I can construct $\Gamma$ either from the original $\gamma_{\mu}$ or the new $\gamma_{\mu}^{\prime}$

$$
\Gamma=\frac{1}{2}\left(\gamma_{1}+\gamma_{2}+\gamma_{3}+\gamma_{4}\right)=\frac{1}{2}\left(\gamma_{1}^{\prime}+\gamma_{2}^{\prime}+\gamma_{3}^{\prime}+\gamma_{4}^{\prime}\right)=\Gamma^{\prime} .
$$

In the free field limit, momentum space again diagonalizes the new action, call it $\bar{D}$,

$$
\bar{D}(p)=2 i \sum_{\mu} \gamma_{\mu}^{\prime} \sin \left(\pi / 2-p_{\mu}\right)
$$

Of course since all I have done is a unitary transformation, the Dirac operators $D$ and $\bar{D}$ are physically equivalent. They also satisfy a complementarity manifested in

$$
D\left(p_{\mu}=\pi / 2\right)=\bar{D}\left(p_{\mu}=0\right)=4 i \Gamma .
$$

This property is crucial to the following construction. 


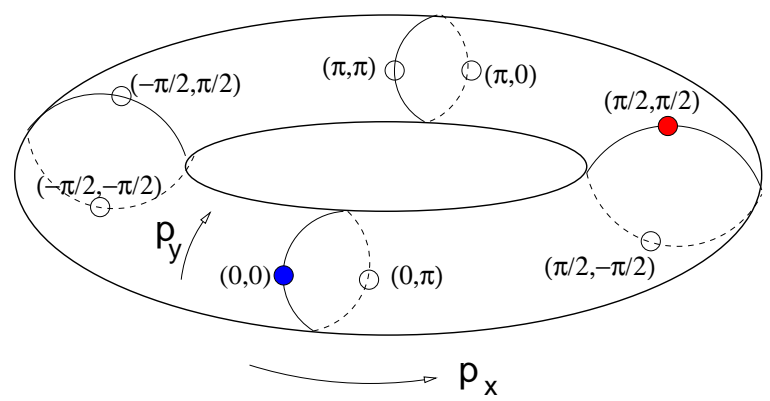

Figure 3: The combination of terms $\mathscr{D}=D+\bar{D}-4 i \Gamma$ leaves only two Fermi points in momentum space. This is the minimal number consistent with the Nielsen-Ninomiya theorem [2].

\section{The minimally doubled action}

I now construct the final action from a linear combination of these two equivalent theories

$$
\mathscr{D}=D+\bar{D}-4 i \Gamma \text {. }
$$

To see how this works, consider the free theory limit in momentum space

$$
\mathscr{D}(p)=2 i \sum_{\mu}\left(\gamma_{\mu} \sin \left(p_{\mu}\right)+\gamma_{\mu}^{\prime} \sin \left(\pi / 2-p_{\mu}\right)\right)-4 i \Gamma .
$$

Going to the Fermi point of the original theory at $p_{\mu} \sim 0$, then the $4 i \Gamma$ term cancels $\bar{D}$. On the other hand, at the Fermi point of $\bar{D}$ occurring at $p_{\mu} \sim \pi / 2$, the $4 i \Gamma$ term cancels $D$. The remarkable feature of this combination is that only these two zeros of $\mathscr{D}(p)$ remain. I give a more detailed proof in Appendix A, but basically at the other zeros of $D, \bar{D}-4 i \Gamma$ is large and at the other zeros of $\bar{D}, D-4 i \Gamma$ is large. Fig. (3) shows the two remaining Fermi points.

Note that each term in Eq. (5.1) anti-commutes with $\gamma_{5}$, maintaining the exact chiral symmetry of the naive action. Thus a finite chiral rotation gives

$$
e^{i \theta \gamma_{5}} \mathscr{D} e^{i \theta \gamma_{5}}=\mathscr{D}
$$

The construction uses different gamma matrices for the two species. A particular consequence is $\gamma_{5}^{\prime}=\Gamma \gamma_{5} \Gamma=-\gamma_{5}$. Under the chiral rotation of Eq. (5.3), the two species behave oppositely. The symmetry is of a non-singlet nature, as expected.

\section{Space time symmetries}

The above construction utilizes the matrix $\Gamma=\frac{1}{2} \sum_{\mu} \gamma_{\mu}$. Had I selected another of the maximally distant points from the zeros of the initial fermion operator, then this relation would be modified with minus signs for some directions. This would give an equivalent theory, but any such choice still involves picking a particular diagonal axis of the fundamental hyper-cubic lattice as special. Here I have chosen the positive major diagonal. For comparison, in Refs. [8,9] the special direction is not a diagonal but rather $x_{4}$, as discussed further in Appendix B.

Having picked a specific diagonal of the fundamental hypercubes, the action is not symmetric under the full hyper-cubic group, but only the subgroup that leaves this direction invariant. This 
subgroup includes $Z_{3}$ rotations that cycle any three positive axes. For example, I might want to cyclicly permute the $x_{1}, x_{2}, x_{3}$ axes. To get the gamma matrices to transform appropriately under such rotations, introduce the matrix (in spinor space)

$$
V=\exp \left(\frac{i \pi}{3 \sqrt{3}}\left(\sigma_{12}+\sigma_{23}+\sigma_{31}\right)\right)
$$

Here I define $\left[\gamma_{\mu}, \gamma_{v}\right]=2 i \sigma_{\mu \nu}$. The combination $V^{\dagger} \gamma_{\mu} V$ cyclicly permutes the first three gamma matrices. Since $[V, \Gamma]=0$, this matrix, along with a corresponding rotation of the gauge fields, realizes a symmetry of the action. Note that $V^{3}=-1$, indicating that a rotation by an angle of $2 \pi$ gives a minus sign, as expected for fermions. Combining such rotations using other axes generates the 12 element tetrahedral subgroup of the hyper-cubic group.

The above rotations generate positive parity permutations of the axes. Introducing negative parity permutations increases the subgroup to 24 elements. For example, the matrix

$$
V=\frac{1}{2 \sqrt{2}}\left(1+i \sigma_{15}\right)\left(1+i \sigma_{21}\right)\left(1+i \sigma_{52}\right)
$$

generates the fermionic part of a rotation that exchanges the $x_{1}$ and $x_{2}$ axes. This transformation flips the sign of $\gamma_{5}$, i.e. $V^{\dagger} \gamma_{5} V=-\gamma_{5}$, emphasizing that it is a negative parity transformation.

In this formalism the natural direction to represent time is the main diagonal $e_{1}+e_{2}+e_{3}+e_{4}$. The combination of time reversal and parity can then be chosen to flip the sign of all axes. A unitarity transformation similar to that relating $D$ and $\bar{D}$ restores the action to its original form, increasing the symmetry group to 48 elements.

Note that charge conjugation symmetry is trivial in this formulation, just being particle hole symmetry. Both the operators $\mathscr{D}$ and the Hermitean combination $\gamma_{5} \mathscr{D}$ have their eigenvalues in opposite sign pairs.

Because of the special treatment of the main diagonal, the effects of interactions can distort lengths along this direction. At each of the Fermi points, this distortion is associated with the dimension five continuum operator $i \bar{\psi} \Gamma \nabla^{2} \psi$. Interactions at finite lattice spacing can result in the gluons and fermions not having the same speed of light. This can be corrected with a renormalization of the $\bar{\psi} \Gamma \psi$ term in the action, as emphasized in Ref. [11]. Nevertheless, the zeros are stable under this distortion because they involve a topologically non-trivial mapping of surfaces of constant action $[2,10]$.

\section{Appendix A: Proof that there are only two zeros of $\mathscr{D}(p)$}

From the definition of $\Gamma$ it is elementary to show that $\gamma_{\mu}^{\prime}=\Gamma-\gamma_{\mu}$ and the properties $\operatorname{Tr} \Gamma \gamma_{\mu}=$ $\operatorname{Tr} \Gamma \gamma_{\mu}^{\prime}=2$. Using trivial trigonometric identities one can deduce

$$
\operatorname{Tr}\left(\gamma_{\mu}-\gamma_{v}\right) \mathscr{D}(p) \sim \sin \left(p_{\mu}-\pi / 4\right)-\sin \left(p_{v}-\pi / 4\right) .
$$

This implies that at any zero $\cos \left(p_{\mu}-\pi / 4\right)= \pm \cos \left(p_{v}-\pi / 4\right)$. But a zero also requires

$$
\operatorname{Tr} \Gamma \mathscr{D}(p)=0 \Rightarrow \sum_{\mu} \cos \left(p_{\mu}-\pi / 4\right)=2 \sqrt{2}>2 .
$$

Thus all these cosines must be positive. Therefore all components of $p_{\mu}$ are equal and either 0 or $\pi / 2$, the two Fermi points of interest. 


\section{Appendix B: Comparison with actions from Karsten and Wilczek}

Two other minimally doubled chiral fermion actions have been presented in Refs. [8, 9]. These actions are in fact equivalent to each other under a unitarity transformation $\psi \rightarrow e^{-i \pi x_{4} / 2} \psi$. For the free case, that action can be written

$$
D=\sum_{\mu=1}^{4} \gamma_{\mu} \sin \left(p_{\mu}\right)+\gamma_{4} \sum_{i=1}^{3}\left(1-\cos \left(p_{i}\right)\right) .
$$

The last term removes all doublers from the naive action except those at $\vec{p}=0$ and $p_{4}=0, \pi$.

The main difference from the action presented here is that $x_{4}$ is now chosen as the special direction. The on site term is proportional to $\gamma_{4}$ instead of $\Gamma$. As before the second species with momentum around $p_{4}=\pi$ uses different effective gamma matrices, $\vec{\gamma}^{\prime}=\vec{\gamma}$ and $\gamma_{4}^{\prime}=-\gamma_{4}$. As with the action from the main text, this gives $\gamma_{5}^{\prime}=-\gamma_{5}$. Again the chiral symmetry is flavored. The symmetry of this system is the subgroup of the hyper-cubic group that preserves the fourth axis up to a sign.

\section{References}

[1] A. Borici, arXiv:0712.4401 [hep-lat].

[2] H. B. Nielsen and M. Ninomiya, Nucl. Phys. B 185, 20 (1981) [Erratum-ibid. B 195, 541 (1982)].

[3] J. B. Kogut and L. Susskind, Phys. Rev. D 11, 395 (1975); L. Susskind, Phys. Rev. D 16, 3031 (1977); H. S. Sharatchandra, H. J. Thun and P. Weisz, Nucl. Phys. B 192, 205 (1981).

[4] K. G. Wilson, in New Phenomena In Subnuclear Physics. Part A. Proceedings of the First Half of the 1975 International School of Subnuclear Physics, Erice, Sicily, July 11 - August 1, 1975, ed.

A. Zichichi, Plenum Press, New York, 1977, p. 69.

[5] P. Hasenfratz and F. Niedermayer, Nucl. Phys. B 414 (1994) 785 [arXiv:hep-lat/9308004];

W. Bietenholz and U. J. Wiese, Nucl. Phys. B 464, 319 (1996) [arXiv:hep-lat/9510026].

[6] D. B. Kaplan, Phys. Lett. B 288 (1992) 342 [arXiv:hep-lat/9206013]; V. Furman and Y. Shamir, Nucl. Phys. B 439, 54 (1995) [arXiv:hep-lat/9405004].

[7] H. Neuberger, Phys. Lett. B 417 (1998) 141 [arXiv:hep-lat/9707022].

[8] L. H. Karsten, Phys. Lett. B 104, 315 (1981).

[9] F. Wilczek, Phys. Rev. Lett. 59, 2397 (1987).

[10] M. Creutz, JHEP 0804, 017 (2008) [arXiv:0712.1201 [hep-lat]].

[11] P. F. Bedaque, M. I. Buchoff, B. C. Tiburzi and A. Walker-Loud, Phys. Lett. B 662, 449 (2008) [arXiv:0801.3361 [hep-lat]]; P. F. Bedaque, M. I. Buchoff, B. C. Tiburzi and A. Walker-Loud, Phys. Rev. D 78, 017502 (2008) [arXiv:0804.1145 [hep-lat]].

[12] K. Cichy, J. Gonzalez Lopez, K. Jansen, A. Kujawa and A. Shindler, Nucl. Phys. B 800, 94 (2008) [arXiv:0802.3637 [hep-lat]].

[13] M. Creutz, Phys. Lett. B 649 (2007) 230 [arXiv:hep-lat/0701018]; M. Creutz, PoS LATTICE2007, 007 (2006) [arXiv:0708.1295 [hep-lat]]. 